\title{
Physical interpretation of the Cosserat mechanics for a collection of atoms
}

\author{
M. SIKON'* \\ Faculty of Mechanics, Institute of Machine Design, Cracow University of Technology, 37 Jana Pawła II St., 31-866 Cracow
}

\begin{abstract}
In this work, the Cosserat medium is analyzed as a set of atoms. These atoms are under the action of a mechanical load. The statistical analysis is preceded by a description of a single atom using classical mechanics and quantum mechanics. The behavior of the atoms in the field generated by mechanical change of the interatomic distance is shown as a phenomenon which can explain the Cosserat mechanics in a continuum..
\end{abstract}

Key words: Cosserat theory, polar atom, mechanical quantum number, statistical mechanics, condensed matter.

\section{Introduction}

Cosserat mechanics [1] (1909) is inspired by the results of experiments conducted by Voigt [2] in 1887. In Cosserat mechanics, we assume that the transmission of mechanical action between two neighboring unit cells of the material by surface element $d F$ is held by force vector $d \boldsymbol{P}$ and moment vector $d \boldsymbol{M}$. Independent of the vector of the force stress $\mathbf{p}=\lim _{d F \rightarrow 0} d \boldsymbol{P} / d F$, the couple stress vector is defined $\mathbf{m}=\lim d \boldsymbol{M} / d F$. A solid deformation is described by a displacement vector $\boldsymbol{u}(x, y, z)$ and by an independent vector of the polar rotation $\varphi(x, y, z)$ [3]. The Cosserat mechanics was elaborated mainly through the theoretical studies. So far we do not know the physical interpretation of the Voigt experiments.

In this work we reference the molecular theory of elasticity. This theory was inspired by Boscovich (1763) [4], and finally formulated by Navier [5] in 1821, while the modern version was developed by Eringen (1967) [6]. Based on the experiments carried out by Voigt, noncentral intermolecular interaction was introduced. Inspired by the work of E. Cosserat and F. Cosserat, Eringen introduced a rotation of the molecule during intermolecular interaction. The physical interpretation of this rotation has not been shown until today.

For analysis of the Cosserat medium in the work presented, the atomic structure of matter was chosen for two reasons:

- the atom is a particle of the granular structure of matter,

- the atom has polar properties.

This paper studies the polar atom, where the mass center and center of the action of the Coulomb forces are not lined up. This disturbed symmetry creates the action arm for interatomic forces. The action arm generates a couple moment, which creates the additional motion of the atom in the form of precession. This precession is considered as a physical interpretation of the theory of micropolar elasticity [6].

*e-mail: sikon@mech.pk.edu.pl

\section{Classical description}

We analyze the set of atoms $k=1,2,3, \ldots N$ in the elementary volume $d V$. In the first approximation, these atoms are analyzed as a set of balls (material points) [7] with vectors of position $\boldsymbol{r}_{k}^{o}$ (initial position) in the Cartesian coordinate system. These balls are at a distance with value $r_{k l}^{o}=\left|\boldsymbol{r}_{l}^{o}-\boldsymbol{r}_{k}^{o}\right|$. When we apply an external mechanical load to the set of atoms $k=1,2,3, \ldots N$ using classical mechanics, we obtain for each atom $k$ the resultant force: $\boldsymbol{P}_{k}=-\sum_{l k}^{N} \nabla_{k} U\left(\boldsymbol{r}_{k t}\right)$, where: $U\left(\boldsymbol{r}_{k l}\right)$ is the diatomic potential of the distance between the atom $k$ and successive atoms $l, l \neq k$, $\nabla$ is the nabla operator. When we take into account the internal structure of the atom $k$ (electrons and nucleons in the shells) [8-9], we assign to the electron/nucleon number $\zeta_{k}=1,2,3, \ldots n_{k}$ a part of the force according to relationship: $\boldsymbol{P}_{k}=\sum_{k=}^{\boldsymbol{P}_{\xi_{k}}}$ and then reduce the force $\boldsymbol{P}_{\zeta_{k}}$ from the central position $C$ (atomic mass center) to the temporary location of the electron/nucleon, the electron/nucleon is additionally loaded by moment $\boldsymbol{M}_{\zeta_{k}}^{\text {couple }}$ (Fig $1)$.



Fig. 1. Reduction of the interatomic interaction on the temporary location of the electron/nucleon

This moment creates precession of the electron/nucleon of angular velocity $\boldsymbol{\omega}_{\zeta_{k}}^{\text {couple }}$. The moment takes the following form:

$$
\boldsymbol{M}_{\zeta_{k}}^{\text {couple }}=\boldsymbol{\omega}_{\zeta_{k}}^{\text {couple }} \times \boldsymbol{j}_{\zeta_{k}},
$$


where $\boldsymbol{j}_{\zeta_{k}}$ is the angular momentum of the electron/nucleon, then the precession energy:

$$
U_{\zeta_{k}}^{\text {couple }}=-\omega_{\zeta_{k}}^{\text {couple }} \cdot \boldsymbol{j}_{\zeta_{k}}
$$

and the angle of precession $\boldsymbol{\varphi}_{\zeta_{k}}^{\text {couple }}$ :

$$
\varphi_{\zeta_{k}}^{\text {couple }}=\operatorname{arcos} \frac{j_{\zeta_{k}}^{\text {couple }}}{j_{\zeta_{k}}}
$$

where $\boldsymbol{j}_{\zeta_{k}}^{\text {couple }}$ is the angular momentum of the precession. So the physical parameters of the electron/nucleon polar elasticity are written as: $\boldsymbol{M}_{\zeta_{k}}^{\text {couple }}, U_{\zeta_{k}}^{\text {couple }}, \boldsymbol{\omega}_{\zeta_{k}}^{\text {couple }}, \boldsymbol{\varphi}_{\zeta_{k}}^{\text {couple }}$, and $\boldsymbol{j}_{\zeta_{k}}^{\text {couple }}$. According to the principle of mechanics, we can formulate the following condition:

$$
\boldsymbol{P}_{\zeta_{k}} \cdot \boldsymbol{\omega}_{\zeta_{k}}^{\text {couple }}=0
$$

where the direction of the force vector $\boldsymbol{P}_{\zeta_{k}}$ and the precession vector $\boldsymbol{\omega}_{\zeta_{k}}^{\text {couple }}$ are perpendicular to one another.

For the whole of the atom $k$ we have to consider the sum of all electrons and nucleons. When the number of electrons in the shells is not equal to $2 n_{e}^{2}\left(n_{e}=1,2,3, \ldots\right.$ is the number of electron shells) and the numbers of nucleons $n_{n}$ in nucleon shells are not equal to magic numbers: $n_{n}=2,8,20,50,82,126, \ldots$, such a model of atom has polar properties and, therefore, is liable to couples and moments. This atom is described by the atomic angular momentum $\boldsymbol{J}_{k}$. The total atomic moment coupled with the mechanical interactions between atoms must be:

$$
\boldsymbol{M}_{k}^{\text {couple }}=\sum_{1}^{n} \boldsymbol{M}_{\zeta_{k}}^{\text {couple }}
$$

where $n$ is the total number of electrons and nucleons.

Using frequency of the atomic precession $\boldsymbol{\omega}_{k}^{\text {couple }}$ and $\boldsymbol{J}_{k}$ we write the atomic couple moment as:

$$
\boldsymbol{M}_{k}^{\text {couple }}=\omega_{k}^{\text {couple }} \times \boldsymbol{J}_{k}
$$

then the atomic couple energy:

$$
U_{k}^{\text {couple }}=-\boldsymbol{\omega}_{k}^{\text {couple }} \cdot \boldsymbol{J}_{k},
$$

and the couple angle of the atomic precession:

$$
\varphi_{k}^{\text {couple }}=\operatorname{arcos} \frac{J_{k}^{\text {couple }}}{J_{k}} .
$$

We fomulate the perpendicularity condition as:

$$
\boldsymbol{P}_{k} \cdot \boldsymbol{\omega}_{k}^{\text {couple }}=0
$$

The physical parameters of the atomic polar elasticity can be denoted as: $\boldsymbol{M}_{k}^{\text {couple }}, U_{k}^{\text {couple }}, \boldsymbol{\omega}_{k}^{\text {couple }}, \boldsymbol{\varphi}_{k}^{\text {couple }}$, and $\boldsymbol{J}_{k}^{\text {couple }}$.

\section{Quantum description}

We assume elimination of the orbital angular momentum of the electron/nucleon, and we define the couple moment $\boldsymbol{M}_{k}^{S}$ in classical mechanics as the cross-product of the angular frequency and spin $\boldsymbol{S}_{k}$ :

$$
\boldsymbol{M}_{k}^{S}=\boldsymbol{\omega}_{k}^{S} \times \boldsymbol{S}_{k}
$$

Equation (10) can be re-written in the form:

$$
\hat{\boldsymbol{M}}_{k}^{S}=\boldsymbol{\Omega}_{k}^{S} \hat{S}_{z}^{k},
$$

where: $\hat{\boldsymbol{M}}_{k}^{S}$ is the operator of the moment $\boldsymbol{M}_{k}^{S}, \Omega_{k}^{S}=\left(\boldsymbol{i} \omega_{y}^{S}-\boldsymbol{j} \omega_{x}^{S}\right)$ $\boldsymbol{i}$, and $\boldsymbol{j}$, are the unit vectors parallel to the $x$, and $y$-axis, and $\hat{S}_{z}^{k}$ is the operator of the component of the spin in $z$-direction (direction of quantization).

According to the quantum theory, the quantum couple moment is determined by solving the eigenvalue equation:

$$
\hat{M}_{k}^{s} \Phi=\mathrm{M}_{k}^{s} \Phi
$$

where $\mathrm{M}_{k}^{S}$ is the eigenvalue of the operator $\hat{M}_{k}^{S}$ and $\Phi$ is the wave function describing the quantum-mechanical state of the atom in the mechanical field. Based on the moment operator (11), the eigenvalue equation for the quantum couple takes the form:

$$
\Omega_{k}^{S} \hat{S}_{z}^{k} \Phi=\mathrm{M}_{k}^{S} \Phi
$$

According to the quantum mechanics, the eigenvalue $\mathbf{M}_{k}^{S}$ is the physical value of the moment action at the nanoscale.

Applying the Pauli operator:

$$
\hat{S}_{z}^{k}=\frac{\hbar}{2}\left(\begin{array}{rr}
1 & 0 \\
0 & -1
\end{array}\right),
$$

where $\hbar=h / 2 \pi$, and $h$ is the Planck constant. Using the wave function:

$$
\Phi= \begin{cases}\Phi \uparrow=\left(\begin{array}{l}
1 \\
0
\end{array}\right) & \text { for } \quad \text { mech }_{S}=\frac{1}{2}, \\
\Phi \downarrow=\left(\begin{array}{l}
0 \\
1
\end{array}\right) & \text { for } \quad \text { mech }_{S}=-\frac{1}{2},\end{cases}
$$

where mech $_{s}$ is the mechanical quantum number (which is analogous to the magnetic quantum number). The eigenvalue equation takes the following form:

$$
\hat{S}_{z}^{k} \Phi=\hbar m e c h_{S} \Phi
$$

which is satisfied as identity. Given the analogy between Eqs. (13) and (16), the eigenvalues for both sets of equations are the same. The solution of the unknown quantum value of the couple moment of the atom $k$ can be written as:

$$
\mathrm{M}_{k}^{S} \Phi=\hbar \Omega_{k}^{S} \text { mech }_{S}
$$


The energy contribution due to the atomic spin $\boldsymbol{S}_{k}$ under mechanical load is written in the classical mechanics in the following form:

$$
U_{k}^{S}=-\omega_{k}^{S} \cdot S_{k}
$$

In the same way as for Eq. (10), we rewrite this in the operator form: $\hat{U}_{k}^{S}=-\boldsymbol{\omega}_{k}^{S} \cdot \hat{\boldsymbol{S}}_{k}$ and we obtain the eigenvalue equation for the atom's energy as follows:

$$
-\boldsymbol{\omega}_{k}^{S} \cdot \hat{\boldsymbol{S}}_{k} \Phi=\mathrm{U}_{k}^{S} \Phi
$$

Equation (19) will now correspond to the Schrödinger equation [10] written in $z$-direction:

$$
-\left(\omega_{z}^{S}\right)_{k} \hat{S}_{z}^{k} \Phi=\mathrm{U}_{k}^{S} \Phi
$$

The operators $\hat{M}_{k}^{s}$ and $\hat{U}_{k}^{s}$ comply with the conditions commutation: $\hat{M}_{k}^{S} \hat{U}_{k}^{S}-\hat{U}_{k}^{S} \hat{M}_{k}^{S}=0$.

We find the quantum value of the precession energy of spin in the same way as before, and finally we obtain:

$$
\mathrm{U}_{k}^{S}=-\left(\omega_{z}^{S}\right) \hbar m e c h_{S} .
$$

Adding the orbital motion, by analogy to solutions (17) and (21), we can formulate the total atomic moment action and total energy of precession in the following form:

$$
\begin{gathered}
\mathrm{M}_{k}^{\text {couple }}=\hbar \Omega_{k}^{\text {couple }} \text { mech }, \\
\mathrm{U}_{k}^{\text {couple }}=-\left(\omega_{z}^{\text {couple }}\right) \hbar \text { mech },
\end{gathered}
$$

where: $\Omega_{k}^{\text {couple }}=\left(\boldsymbol{i} \omega_{y}^{\text {couple }}-\boldsymbol{j} \omega_{x}^{\text {couple }}\right)_{k}$,

$$
\text { mech }=-J,-J+1, \ldots, 0, \ldots, J-1, J
$$

and $J$ is the total atomic quantum number. Introducing $\mathrm{J}_{k}=$ $\hbar \sqrt{J(J+1)}$ and $\mathrm{J}_{k}^{\text {couple }}=\hbar$ mech, [11], we obtain the quantum formula for polar rotation of the atom:

$$
\varphi_{k}^{\text {couple }}=\operatorname{arcos} \frac{\text { mech }}{\sqrt{J(J+1)}} .
$$

\section{Statistical description}

We describe the probabilistic space as a set of atoms $k=1,2,3, \ldots, N_{F}$ in the elementary area $d F$. The set of the mechanical quantum numbers mech $=-J,-J+1, \ldots, 0, \ldots, J-1, J$ comprises random numbers. The elementary events are defined as: $\boldsymbol{M}_{k}^{\text {couple }}, U_{k}^{\text {couple }}$, $\boldsymbol{\omega}_{k}^{\text {couple }}, \boldsymbol{\varphi}_{k}^{\text {couple }}$, and $\boldsymbol{J}_{k}^{\text {couple }}$. We write the random variables:

$$
\begin{gathered}
\mathrm{M}_{k}^{\text {couple }}=\hbar \Omega_{k}^{\text {couple }} \text { mech, } \\
\mathrm{U}_{k}^{\text {couple }}=-\left(\omega_{z}^{\text {couple }}\right) \hbar m e c h, \\
\mathrm{~J}_{k}^{\text {couple }}=\hbar m e c h,
\end{gathered}
$$

$$
\varphi_{k}^{\text {couple }}=\operatorname{arcos} \frac{\text { mech }}{\sqrt{J(J+1)}},
$$

which are functions of the mechanical quantum numbers mech. Based on the fundamental assumption of statistical mechanics and Boltzmann distribution [12], we obtain the canonical distribution:

$$
\Pi(\text { mech })=\frac{\exp \left[-\frac{\mathrm{U}(\text { mech })}{\mathrm{k} T}\right]}{\sum_{\text {mech }=-J}^{\text {mech }=J}\left[-\frac{\mathrm{U}(\text { mech })}{\mathrm{k} T}\right]},
$$

where $\mathrm{k}$ is the Boltzmann constant, $T$ is temperature. The probabilistic space concerns the atoms where interatomic actions are almost equal: $\boldsymbol{P}_{k} \approx \boldsymbol{P}$, and then we write: $\boldsymbol{\omega}_{k}^{\text {couple }} \approx \boldsymbol{\omega}^{\text {couple }}$, $\boldsymbol{\Omega}_{k}^{\text {couple }} \approx \boldsymbol{\Omega}^{\text {couple }}$. Now we can write the average values of the Cosserat parameters in a canonical distribution:

$$
\begin{array}{r}
\left\langle\mathrm{M}_{k}^{\text {couple }}\right\rangle=\sum_{m e c h=-J}^{m e c h=J} \mathrm{M}_{k}^{\text {couple }} \Pi=\hbar \Omega_{k}^{\text {couple }} J B_{J}, \\
\left\langle\mathrm{U}_{k}^{\text {couple }}\right\rangle=\sum_{m e c h=-J}^{m e c h=J} \mathrm{U}_{k}^{\text {couple }} \Pi=\hbar \omega_{z}^{\text {couple }} J B_{J}, \\
\left\langle\mathrm{~J}_{k}^{\text {couple }}\right\rangle=\sum_{m e c h=-J}^{m e c h=J} \mathrm{~J}_{k}^{\text {couple }} \Pi=\hbar J B_{J}, \\
\left\langle\cos \varphi_{k}^{\text {couple }}\right\rangle=\sum_{m e c h=-J}^{m} \frac{m e c h}{\sqrt{J(J+1)}} \Pi=\frac{J}{\sqrt{J(J+1)}} B_{J},
\end{array}
$$

where:

$B_{J}=\left[\frac{2 J+1}{2 J} \operatorname{ctgh}\left(\frac{2 J+1}{2} \frac{\omega_{z}^{\text {couple }} \hbar}{\mathrm{k} T}\right)-\frac{1}{2 J} \operatorname{ctgh}\left(\frac{\omega_{z}^{\text {couple }} \hbar}{2 \mathrm{k} T}\right)\right]$

is a function analogous to the Brillouin function [11].

Using the concentration of atoms $\mathrm{N}=\mathrm{N}_{F} / d F$, we obtain the statistical couple moment, energy of precession, precession moment of momentum and angle of precession in the form:

$$
\begin{gathered}
\mathbf{m}^{\text {couple }}=\mathrm{N} \hbar \Omega_{k}^{\text {couple }} J B_{J}, \\
U^{\text {couple }}=\mathrm{N} \hbar \omega_{z}^{\text {couple }} J B_{J}, \\
J^{\text {couple }}=\hbar J B_{J}, \\
\varphi^{\text {couple }}=\arccos \frac{J}{\sqrt{J(J+1)}} B_{J} .
\end{gathered}
$$

The statistical couple moment $\mathbf{m}^{\text {couple }}$ and the angle of precession $\varphi^{\text {couple }}$ correspond to the vector of the moment stress $\mathbf{m}$ and the polar rotation $\varphi$ introduced in Cosserat mechanics. 
In room temperature, when $\frac{\omega_{z}^{\text {couple }} \hbar}{\mathrm{k} T}<<1$, hyperbolic cotangent in $B_{J}$ function takes the form of a power series. Leaving only the first two terms, we obtain:

$$
\begin{aligned}
\mathbf{m}^{\text {couple }} & =\mathrm{N} \hbar^{2} \Omega^{\text {couple }} J(J+1) \frac{\omega_{z}^{\text {couple }}}{3 \mathrm{k} T}, \\
U^{\text {couple }} & =\mathrm{N} \hbar^{2} J(J+1) \frac{\left(\omega_{z}^{\text {couple }}\right)^{2}}{3 \mathrm{k} T}, \\
\boldsymbol{J}^{\text {couple }} & =\mathrm{N} \hbar^{2} J(J+1) \frac{\omega_{z}^{\text {couple }}}{3 \mathrm{k} T}, \\
\varphi^{\text {couple }} & =\arccos \sqrt{J(J+1)} \frac{\omega_{z}^{\text {couple }} \hbar}{3 \mathrm{k} T} .
\end{aligned}
$$

In the particular case when $J=\frac{1}{2}$ formulas (39), .., (41) we write:

$$
\begin{gathered}
\mathbf{m}^{\text {couple }}=\mathrm{N} \hbar^{2} \Omega^{\text {couple }} \frac{\omega_{z}^{\text {couple }}}{4 \mathrm{k} T}, \\
U^{\text {couple }}=\mathrm{N} \hbar^{2} \frac{\left(\omega_{z}^{\text {couple }}\right)^{2}}{4 \mathrm{k} T}, \\
\boldsymbol{J}^{\text {couple }}=\mathrm{N} \hbar^{2} \frac{\omega_{z}^{\text {couple }}}{4 \mathrm{k} T}, \\
\varphi^{\text {couple }}=\arccos \frac{\sqrt{3}}{6} \frac{\omega_{z}^{\text {couple }} \hbar}{3 \mathrm{k} T} .
\end{gathered}
$$

In very low temperature and very strong mechanical interactions between the atoms (in elastic range), where

$$
T<1 K, \quad \frac{\omega_{z}^{\text {couple }} \hbar}{\mathrm{k} T}>>1,
$$

we obtain:

$$
\begin{gathered}
\lim _{T<1 K, \frac{\omega_{z}^{\text {couple }} \hbar}{\mathrm{k} T} \gg 1} \operatorname{ctgh}\left(\frac{2 \mathrm{~J}+1}{2} \frac{\omega_{z}^{\text {couple }} \hbar}{\mathrm{k} T}\right)=1, \\
\lim _{T<1 K, \frac{\omega_{z}^{\text {couple }} \hbar}{\mathrm{k} T} \gg 1} \operatorname{ctgh}\left(\frac{\omega_{z}^{\text {couple }} \hbar}{\mathrm{k} T}\right)=1,
\end{gathered}
$$

and formulas (35-38) take the following forms:

$$
\begin{gathered}
\mathbf{m}^{\text {couple }}=\mathrm{N} \hbar \Omega^{\text {couple }} J, \\
U^{\text {couple }}=\mathrm{N} \hbar \omega_{z}^{\text {couple }} J, \\
J^{\text {couple }}=\mathrm{N} \hbar J, \\
\varphi^{\text {couple }}=\arccos \frac{J}{\sqrt{J(J+1)}},
\end{gathered}
$$

Now, for the particular case when $J=\frac{1}{2}$ :

$$
\mathbf{m}^{\text {couple }}=\mathrm{N} \frac{\hbar \Omega^{\text {couple }}}{2},
$$

$$
\begin{gathered}
U^{\text {couple }}=\mathrm{N} \frac{\hbar \omega_{z}^{\text {couple }}}{2}, \\
J^{\text {couple }}=\mathrm{N} \frac{\hbar}{2}, \\
\varphi^{\text {couple }}=\left\{\begin{array}{l}
54,7^{\circ} \\
125,3^{\circ}
\end{array}\right.
\end{gathered}
$$

\section{Polarization of the atoms in a mechanical field}

We define the macroscopic vector of the moment of momentum on the surface $d F$ in the form:

$$
\boldsymbol{J}=\frac{1}{d F} \sum_{k=1}^{N_{F}} \boldsymbol{J}_{k} .
$$

When the system of the atoms $k=1,2,3, \ldots, N_{F}$ is not loaded, the vectors of the atomic moment of momentum $\boldsymbol{J}_{k}$ are oriented in space in a completely disordered way and the total macroscopic moment of momentum is equal to zero $\boldsymbol{J}=0$. At the time of application of the external load, the macroscopic vector $\boldsymbol{J}$ increases from zero to the value $\boldsymbol{J}^{\text {couple }}$ as on formula (37) and is set in a direction perpendicular to the average direction of the interatomic action. This process lasts for the time $T$, described by the formula:

$$
\frac{d \boldsymbol{J}}{d t}=\frac{\boldsymbol{J}^{\text {couple }}-\boldsymbol{J}}{T} .
$$

Solving equation (59), [11], we can write the dependence of the macroscopic moment of momentum on the time:

$$
\boldsymbol{J}(t)=\boldsymbol{J}^{\text {couple }}\left(1-e^{-\frac{t}{T}}\right)
$$

When a Cosserat medium is subjected to mechanical loads, the polarization of the macroscopic moment of momentum $\boldsymbol{J}$ occurs. This polarization can be characterized by the statistical angle of precession $\varphi^{\text {couple }}$ written in formula (38). During this process the mechanical energy:

$$
U^{\text {couple }}(t)=-\boldsymbol{\omega}^{\text {couple }} \cdot \boldsymbol{J}(t),
$$

decreases until the macroscopic moment of momentum $\boldsymbol{J}$ reaches a value $\boldsymbol{J}^{\text {couple }}$ corresponding to the new state of equilibrium.

\section{Conclusion}

Voigt could not explain the results of his experiments because knowledge about the atom was not sufficient at that time (Thomson model). We also do not have such a description in contemporary works of interatomic interactions, even though the planetary model of the atom has been present in physics 
since 1913. The planetary structure of the atom is not taken into account in the mechanical interactions in the first studies using quantum mechanics $[10,14]$, and is not taken into account in attempts to provide the mechanical stress and force at the quantum level [15].

As was shown in the above analysis, the general phenomenon which can explain the Cosserat mechanics by the scale electron/nucleon is the precession of the electron/nucleon shell in the field induced by mechanical change of the interatomic distance. The atom precession occurs as a result of the asymmetrical construction of the atom. For a set of atoms we can describe phenomena of the polarization of atoms. The results of the analysis in a different scale correspond to the basic of the condensed matter enunciated by P. W. Anderson [13].
The physical interpretation of the Cosserat mechanics (moment action, energy of precession, moment of momentum of precession and angle of precession) is derived on the basis of the electron/nucleon structure of the matter, the quantum properties of the matter and the Boltzmann distribution. This analysis can explain the results of the Voigt experiment, the rotation of the molecule introduced ad hoc in the micropolar elasticity and the polar properties of the materials introduced ad hoc in the nonsymmetrical elasticity.

The quantum volume of the energy association with disturbance of the electron/nucleon in the field generated by mechanical action can become an inspiration to carry on experiments in resonance spectrometers EPR and NMR in order to confirm the above theory.

Appendix. The average values of the $\cos \varphi_{k}^{\text {couple }}$ for atoms $k=1,2,3, \ldots, N_{F}$ location on interaction area in canonical distribution $\Pi$ take the following form:

$$
\left\langle\cos \varphi_{k}^{\text {couple }}\right\rangle=\sum_{m e c h=-J}^{\text {mech=J }} \frac{m e c h}{\sqrt{J(J+1)}} \Pi=\frac{J}{\sqrt{J(J+1)}} \frac{\sum_{m e c h=-J}^{m e c h=J} \operatorname{mech} \exp \left[\frac{\omega_{z}^{\text {couple }} \hbar m e c h}{\mathrm{k} T}\right]}{\sum_{m e c h=-J}^{m e c h=J} \exp \left[\frac{\omega_{z}^{\text {couple }} \hbar m e c h}{\mathrm{k} T}\right]} .
$$

Expression $\frac{\omega_{z}^{\text {couple }} \hbar}{\mathrm{k} T}$ is a variable, so formula (A1) can be written as:

$$
\left\langle\cos \varphi_{k}^{\text {couple }}\right\rangle=\frac{J}{\sqrt{J(J+1)}} \frac{d}{d\left(\frac{\omega_{z}^{\text {couple }} \hbar}{\mathrm{k} T}\right)} \ln \sum_{m e c h=-J}^{\text {mech=J }} \exp \left[\frac{\omega_{z}^{\text {couple }} \hbar m e c h}{\mathrm{k} T}\right]
$$

The sum in (A2) takes the form:

$$
\begin{gathered}
\sum_{m e c h=-J}^{\text {mech }=J} \exp \left[\frac{\omega_{z}^{\text {couple }} \hbar m e c h}{\mathrm{k} T}\right]=\exp \left[\frac{\omega_{z}^{\text {couple }} \hbar(-J)}{\mathrm{k} T}\right]+\exp \left\{\frac{\omega_{z}^{\text {couple }} \hbar[-(J-1)]}{\mathrm{k} T}\right\}+, \ldots \\
\ldots,+\exp \left[\frac{\omega_{z}^{\text {couple }} \hbar(J-1)}{\mathrm{k} T}\right]+\exp \left\{\frac{\omega_{z}^{\text {couple }} \hbar J}{\mathrm{k} T}\right\}
\end{gathered}
$$

and after transformation:

$$
\begin{gathered}
\sum_{\text {mech }=-J}^{\text {mech }=J} \exp \left[\frac{\omega_{z}^{\text {couple }} \hbar m e c h}{\mathrm{k} T}\right]=\exp \left[\frac{\omega_{z}^{\text {couple }} \hbar(-J)}{\mathrm{k} T}\right]\left\{1+\exp \left(\frac{\omega_{z}^{\text {couple }} \hbar}{\mathrm{k} T}\right)+, \ldots\right. \\
\left.\ldots,+\exp \left[\frac{\omega_{z}^{\text {couple }} \hbar(2 \mathrm{~J}-1)}{\mathrm{k} T}\right]+\exp \left(\frac{\omega_{z}^{\text {couple }} \hbar 2 J}{\mathrm{k} T}\right)\right\}
\end{gathered}
$$

Using the sum of a power series formula (A4) we write in the form:

$$
\sum_{m e c h=-J}^{\operatorname{mech}=J} \exp \left[\frac{\omega_{z}^{\text {couple }} \hbar m e c h}{\mathrm{k} T}\right]=\exp \left[\frac{\omega_{z}^{\text {couple }} \hbar(-J)}{\mathrm{k} T}\right] \frac{1-\exp \left[\frac{\omega_{z}^{\text {couple }} \hbar(2 J+1)}{\mathrm{k} T}\right]}{1-\exp \left[\frac{\omega_{z}^{\text {couple }} \hbar}{\mathrm{k} T}\right]},
$$


and after transformation:

$$
\sum_{m e c h=-J}^{\text {mech }=J} \exp \left[\frac{\omega_{z}^{\text {couple }} \hbar m e c h}{\mathrm{k} T}\right]=\exp \left[\frac{\omega_{z}^{\text {couple }} \hbar(-J)}{\mathrm{k} T}\right] \frac{1-\exp \left[\frac{\omega_{z}^{\text {couple }} \hbar(2 J+1)}{\mathrm{k} T}\right]}{1-\exp \left[\frac{\omega_{z}^{\text {couple }} \hbar}{\mathrm{k} T}\right]} .
$$

Substituting (A6) to formula (A2) and counting derivative, we finally obtain:

$$
\left\langle\cos \varphi_{k}^{\text {couple }}\right\rangle=\frac{J}{\sqrt{J(J+1)}}\left[\frac{2 J+1}{2 J} \operatorname{ctgh}\left(\frac{2 J+1}{2} \frac{\omega_{z}^{\text {couple }} \hbar}{\mathrm{k} T}\right)-\frac{1}{2 J} \operatorname{ctgh}\left(\frac{\omega_{z}^{\text {couple }} \hbar}{2 \mathrm{k} T}\right)\right] .
$$

\section{REFERENCES}

[1] E. Cosserat and F.Cosserat, Théorie des corps déformables, A. Hermann, Paris 1909.

[2] W. Voigt, Theoretishe Studien über die Elastizitätsverhältnisse der Kristalle, Abh. Ges.Wiss. Göttning, 43, 1887.

[3] W. Nowacki., Theory of the nonsymmetrical elasticity, Polish Academy of Sciences, PWN, Warsaw 1981.

[4] R. J. Boscovich, Philosophie naturalis, Venezia 1763.

[5] C. L. Navier, Mémoire sur les lois de l'éqilibre mouvement des corps solides élastiques, Mém. Inst. Natl., 1824.

[6] A. C. Eringen, Theory of micropolar elasticity, Princeton University, Princeton, New Jersey, 1967.

[7] M. P. Allen, Introduction to molecular dynamics simulation, John von Neumann Inst. For Comput., vol. 23, 2004.

[8] N. Bohr, Studies on the Electron Theory of Metals, University of Copenhagen, Copenhagen 1911.
[9] J. H. Bartlett, Nuclear structure, Nature, 130, 165, 1932.

[10] E. Schrödinger, Collected Papers on Wave Mechanics, Blackie, Glasgow, 1928 .

[11] C. Kittel, Introduction to Solid State Physics, 8th ed. Jon Willey \& Sons, Inc., New York 2005.

[12] L. D. Landau J. M. Lifszyc, Statistical physics, PWN, 2011.

[13] P. W. Anderson, Basic Notions of Condensed Matter Physics. Reading: Addison-Wesley, 1997, ISBN 0-201-32830-5.

[14] R. P. Feynman, R. B. Leighton and M. Sands, Feynman Lecture on Physics, Volume III, Reading, MA: Addison-Wesley, 1963.

[15] O. H. Nielsen, R. M. Martin, Quantum-mechanical theory of stress and force, Physical Review B, Volume 32, Nr 6, 1985.

[16] M. Sikon, Analysis of the Cosserat medium on the basis of the atomic structure of the matter, Monograph, Publ. Cracow University of Technology, Cracow 2012. 